\title{
AN INVERSE PROBLEM IN DIFFERENTIAL EQUATIONS*
}

BY R. E. LANGER

1. Introduction. The differential equation as a tool requires no introduction to either the mathematician or the applied scientist. Problems in endless variety are continually solved through this medium, the process almost invariably beginning with an epitome of the problem's essential characteristics in the form of a differential equation, which is thus determined explicitly both as to its structure and its coefficients, and proceeding thence to a deduction of the form or properties of a suitable solving function.

The present note is devoted to a problem in which this customary order of events is in large measure reversed. The formulation of the problem yields in this case the structural form of a differential equation, and beyond this the existence of a solution which satisfies certain specified conditions. From these data the determination of the equation itself, that is, of its coefficient function, is required and constitutes the solution of the problem.

2. The Physical Problem. $t$ In the investigation of shallow geological structures, and in the study of the electrical resistivity of the earth's crust at depths below the surface, an appropriate experimental procedure centers around the supply of a direct electric current through a small electrode to the surface of the earth. The electrical potentials which result at the surface of the earth are measurable at all distances from the electrode, and constitute entirely the immediately obtainable data. From them it is desired to compute, if possible, the conductivity of the earth below as a function of the depth.

With the idealizations involved in regarding the conductivity as a differentiable point function depending upon the depth alone, and in taking the ground as a horizontally uniform in-

* Presented to the Society, April 14, 1933.

† The problem and its formulation as outlined in this section are due to Professor L. B. Slichter of the Massachusetts Institute of Technology. A complete and detailed discussion of it is given in a geophysical paper by L. B. Slichter, in Physics, vol. 4, Sept., 1933. 
finite half-space, the problem may be formulated in the following way. Let $x$, the depth, and $\rho$, the horizontal distance from the electrode, be taken as cylindrical coordinates with origin at the electrode. The electrical potential $\phi(\rho, x)$ may be shown then to satisfy the differential equation

$$
\sigma(x)\left[\frac{\partial^{2} \phi}{\partial \rho^{2}}+\frac{1}{\rho} \frac{\partial \phi}{\partial \rho}+\frac{\partial^{2} \phi}{\partial x^{2}}\right]+\frac{d \sigma(x)}{d x} \cdot \frac{\partial \phi}{\partial x}=0,
$$

in which $\sigma(x)$ denotes the earth's conductivity. The substitution

$$
\phi(\rho, x)=U(\rho) u(x)
$$

separates the variables and resolves the equation into the components

$$
\begin{aligned}
U^{\prime \prime}+\rho^{-1} U^{\prime}+\lambda^{2} U & =0, \\
\left\{\sigma u^{\prime}\right\}^{\prime}-\lambda^{2} \sigma u & =0 .
\end{aligned}
$$

The first of these is a Bessel equation, and, since the potential is to remain finite and vanish at infinity like the reciprocal of the distance, it must be concluded that $U(\rho) \equiv J_{0}(\lambda \rho)$.

The second component equation is of the Sturm-Liouville type, and since $\sigma(x)$ is positive, its solutions are of exponential form. Let $u_{1}(x, \lambda)$ denote a solution which is positive, and, as a function of $x$, monotonically decreasing. The condition that $\partial \phi / \partial x$ vanish everywhere at the surface except at the electrode leads by familiar reasoning to the formula

$$
\phi(\rho, x)=\frac{-c}{2 \pi a \sigma(0)} \int_{0}^{\infty} \frac{u_{1}(x, \lambda)}{u^{\prime}(0, \lambda)} J_{0}(\lambda \rho) \sin \lambda a d \lambda,
$$

in which $c$ is the current, and $a$ the radius of the electrode. In terms of the abbreviation

$$
\Omega(\lambda) \equiv-\lambda u_{1}(0, \lambda) / u_{1}^{\prime}(0, \lambda),
$$

the surface potentials are accordingly given by

$$
\phi(\rho, 0)=\frac{c}{2 \pi \sigma(0)} \int_{0}^{\infty} \Omega(\lambda) \frac{\sin \lambda a}{\lambda a} J_{0}(\lambda \rho) d \lambda,
$$

a relation which by the Fourier-Bessel integral theorem may be inverted into the form 


$$
\Omega(\lambda)\left[c(\sin \lambda a) /(2 \pi a) \lambda^{2} \sigma(0)\right]=\int_{0}^{\infty} \phi(\rho, 0) J_{0}(\lambda \rho) \rho d \rho .
$$

The formulas thus derived reveal the significant fact that the functions $\Omega(\lambda)$ and $\phi(\rho, 0)$ are each uniquely determined by the other, and hence that the information embodied in the surface potential data is completely embraced in the function $\Omega(\lambda)$ when the latter is given for $0<\lambda<\infty$. It is to be shown how from these data the conductivity function $\sigma(x)$ may be computed.

3. The Mathematical Problem. The considerations sketched in brief above may be looked upon as having crystallized the physical problem into the following somewhat idealized mathematical one.

On some interval $0 \leqq x \leqq h$ a certain function $\sigma(x)$ is known to be analytic and positive. Beyond this it is known that the differential equation

$$
\frac{d}{d x}\left\{\sigma(x) \frac{d u}{d x}\right\}-\lambda^{2} \sigma(x) u=0
$$

possesses a solution $u_{1}(x, \lambda)$ which has the properties:

(i) that for $\lambda$ on the range $(0, \infty)$ and $x$ on the interval $(0, h)$ the relations

$$
u_{1}(x, \lambda)>0, u_{1}^{\prime}(x, \lambda)<0,
$$

are satisfied; while

(ii) at $x=0$, the boundary condition

$$
\Omega(\lambda) u_{1}^{\prime}(0, \lambda)+\lambda u_{1}(0, \lambda)=0
$$

is fulfilled, $\Omega(\lambda)$ being a function which is compatible with the preceding hypothesis, and which is known and given for $0<\lambda<\infty$. The function $\sigma(x)$ is to be computed.

A process for the desired computation may be deduced as follows. Let the equation (1) be written in the form

$$
u^{\prime \prime}(x, \lambda)+\frac{\sigma^{\prime}(x)}{\sigma(x)} u^{\prime}(x, \lambda)-\lambda^{2} u(x, \lambda)=0 .
$$

It is then readily seen to possess a fundamental set of solutions which are represented asymptotically (as to $\lambda$ ) by a pair of expressions 
in which

$$
e^{ \pm \lambda x} S(x, \pm \lambda)
$$

$$
S(x, \lambda)=\sigma^{-1 / 2}(x)\left[1+\sum_{n=1}^{\infty} \alpha_{n}(x) \lambda^{-n}\right] .
$$

Hence the solution which satisfies the condition (3), and includes a suitable factor independent of $x$, is representable by a formula

$$
u_{1}(x, \lambda) \sim e^{-\lambda x} S(x,-\lambda)+\frac{\Omega(\lambda)-1}{\Omega(\lambda)+1} e^{\lambda x} S(x, \lambda) .
$$

Now unless the first term on the right of this expression is dominant for all values of $x$ on $(0, h)$, the one or the other of the conditions (2) will inevitably be violated when $\lambda$ is sufficiently large. It follows, therefore, that

$$
u_{1}(x, \lambda) \sim e^{-\lambda x} S(x,-\lambda),
$$

a form which on substitution into the condition (3) is found to impose upon the function $\Omega(\lambda)$ a condition of compatibility to the effect that it admit of a representation

$$
\Omega(\lambda) \sim 1+\sum_{n=1}^{\infty} \omega_{n} \lambda^{-n}
$$

Since by hypothesis the function $\Omega(\lambda)$ is given, the infinite set of constants $\omega_{n},(n=1,2,3, \cdots)$, is to be considered as known.

Let the function $v(x, \lambda)$ be defined by the formula

$$
v(x, \lambda)=-\lambda u_{1}(x, \lambda) / u_{1}^{\prime}(x, \lambda) .
$$

It is then found on the one hand in virtue of (1a) to satisfy the Riccati equation

$$
v^{\prime}(x, \lambda)-\frac{\sigma^{\prime}(x)}{\sigma(x)} v(x, \lambda)-\lambda v^{2}(x, \lambda)+\lambda=0,
$$

and on the other hand, in virtue of (3), (4) and (5), to be asymptotically representable in a form

$$
v(x, \lambda) \sim 1+\sum_{n=1}^{\infty} v_{n}(x) \lambda^{-n},
$$

the coefficients $v_{n}(x)$ being analytic on $(0, h)$, and satisfying the boundary relations 


$$
v_{n}(0)=\omega_{n}, \quad(n=1,2,3, \cdots) .
$$

A relation

$$
v^{-1}(x) \sim 1+\sum_{n=1}^{\infty} r_{n}(x) \lambda^{-n}
$$

then follows, the coefficients being related to those of the series (8) by the recurrence formulas*

$$
r_{n}(x)=-\sum_{j=1}^{n-1} v_{j}(x) r_{n-j}(x)-v_{n}(x), \quad(n=1,2,3, \cdots) .
$$

If the equation ( 7 ) is now written in the form

$$
\frac{\sigma^{\prime}(x)}{\sigma(x)}=-\lambda v(x, \lambda)+\frac{v^{\prime}(x, \lambda)+\lambda}{v(x, \lambda)},
$$

and the series (8) and (8a) are formally substituted in it, it is found as a result that

$$
\begin{aligned}
\frac{\sigma^{\prime}(x)}{\sigma(x)} \sim-v_{1}(x)+ & r_{1}(x) \\
& +\sum_{n=1}^{\infty} \lambda^{-n}\left[v_{n}^{\prime}+r_{n+1}-v_{n+1}+\sum_{j=1}^{n-1} v_{j}^{\prime} r_{n-j}\right] .
\end{aligned}
$$

The function $\sigma(x)$, however, does not depend upon $\lambda$. Hence it must be concluded that

$$
\frac{\sigma^{\prime}(x)}{\sigma(x)}=-v_{1}(x)+r_{1}(x)
$$

and

$$
\sum_{j=1}^{n-1} v_{j}^{\prime} r_{n-j}+v_{n}^{\prime}=v_{n+1}-r_{n+1}, \quad(n=1,2,3, \cdots) .
$$

Of the equations (12) the first $p$ in number when taken together constitute a linear algebraic system for the unknowns $v_{1}^{\prime}, v_{2}^{\prime}, \cdots, v_{p}^{\prime}$. The system has a determinant of value unity and on solution yields the formulas

* In this as well as in subsequent formulas an indicated sum is to be considered as zero if the upper index of summation is less than the lower one. 


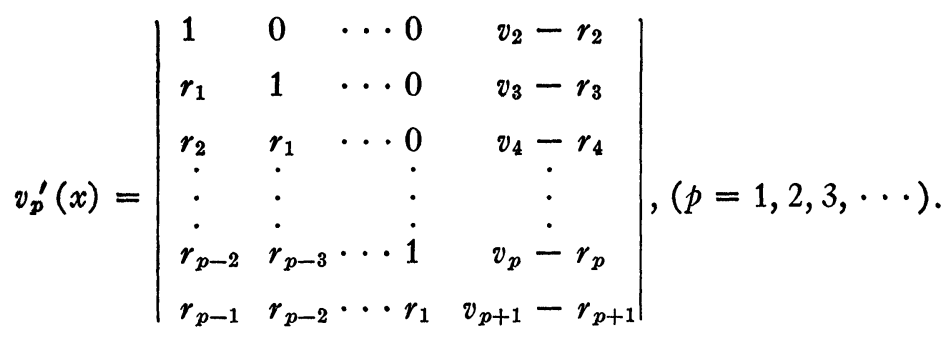

These formulas may be materially simplified as follows. The iteration of the formula (10) yields the relations

$$
-r_{n}=\sum_{j=2}^{n-1}\left(v_{j}-v_{1} v_{j-1}\right) r_{n-j}+\left(v_{n}-v_{1} v_{n-1}\right),
$$

from which it may be seen that if to the last column in the determinant (13) there is added the combination

$$
-\sum_{j=1}^{p-1}\left(v_{j+1}-v_{1} v_{j}\right) \text { times the } j \text { th column, }
$$

the effect is formally to replace the elements $r_{2}, r_{3}, \cdots, r_{p}$ by zeros and in the case of the last row to replace $-r_{p+1}$ by $v_{p+1}-v_{1} v_{p}$. In precisely the same way it will be seen upon reference to the formulas (10), that if to the first column in (13) there is added the combination

$$
\sum_{j=2}^{p-1} v_{j-1} \text { times the } j \text { th column, }
$$

the formal effect is to replace the elements $r_{1}, r_{2}, \cdots, r_{p-2}$ by zeros, and in the case of the last row to replace $r_{p-1}$ by $-v_{p-1}$. Similar reductions of the remaining columns may likewise be made, the formula (13) being reduced in consequence to the form

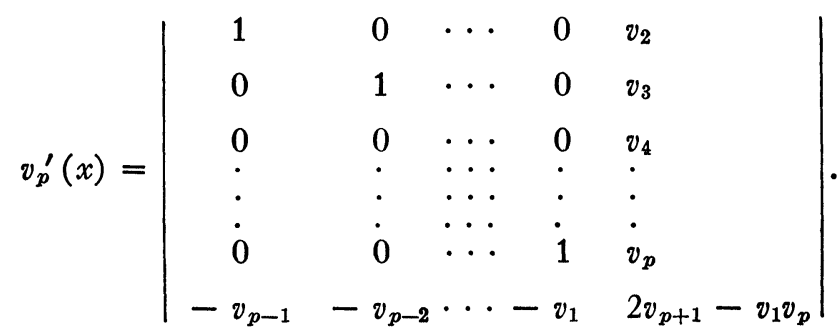


If in this, finally, there is added to the last row the combination

$$
\sum_{j=1}^{p-1} v_{p-j} \text { times the } j \text { th row, }
$$

the determinant disintegrates into the formulas

in which

$$
\begin{array}{ll}
v_{1}^{\prime}(x) & =f_{1}\left(v_{1}, v_{2}\right), \\
v_{n}^{\prime}(x) & =\phi_{n}(v),
\end{array} \quad(n=2,3,4, \cdots),
$$

$$
\begin{aligned}
f_{1}\left(v_{1}, v_{2}\right) & =2 v_{2}(x)-v_{1}^{2}(x), \\
\psi_{n}(v) & =2 v_{n+1}(x)+\sum_{j=1}^{n-2} v_{j+1}(x) v_{n-j}(x) .
\end{aligned}
$$

Let the functions $f_{2}, f_{3}, \cdots$, be defined successively by the recurrence formula

$$
f_{n}\left(v_{1}, v_{2}, \cdots, v_{n+1}\right)=\sum_{i=1}^{n} \phi_{i}(v) \frac{\partial f_{n-1}}{\partial v_{i}} .
$$

Then it is readily seen that repeated differentiation of the first of formulas (14), and substitution from the remaining formulas, gives the expressions

$$
\frac{d^{n} v_{1}(x)}{d x^{n}}=f_{n}\left(v_{1}, v_{2}, \cdots, v_{n+1}\right), \quad(n=1,2,3, \cdots),
$$

for the derivatives of the function $v_{1}(x)$.

For general values of $x$ the functions involved in the right member of (17) are not known. They are given, however, for $x=0$ by (9), whence

$$
\left.\frac{d^{n} v_{1}}{d x}\right]_{x=0}=f_{n}\left(\omega_{1}, \omega_{2}, \cdots, \omega_{n+1}\right),(n=1,2,3, \cdots) .
$$

With these values available the MacLaurin expansion of the function $v_{1}(x)$ is computable, and since the first of the formulas (10) gives to (11) the form

$$
\frac{\sigma^{\prime}(x)}{\sigma(x)}=-2 v_{1}(x)
$$

the desired computation of the function $\sigma(x)$ has been accomplished.

The University of Wisconsin 